\title{
The Effect of Ultrasound Pre-treatment on the hot air Drying Rate of Carrots
}

\author{
Yaxiang Bai \\ School of Science, Dalian Ocean University \\ Dalian Ocean University \\ Liaoning Province, China \\ Byx0671@163.com \\ Yuan Huo \\ School of Science, Dalian Ocean University \\ Dalian Ocean University \\ Liaoning Province, China \\ 653785294@qq.com
}

\author{
Bin Kang \\ School of Science, Dalian Ocean University \\ Dalian Ocean University \\ Liaoning Province, China \\ 499238181@qq.com \\ Hang Sun \\ School of Science, Dalian Ocean University \\ Dalian Ocean University \\ Liaoning Province, China \\ 550205059 @q.com
}

\begin{abstract}
In order to improve the drying rate and to reduce the energy consumption of vegetables and fruits, carrots chips are treated with ultrasonic with different frequency, time, and different temperature. The hot air drying rate of treated carrots chips is determined and, meanwhile, several quality parameters, such as the shrinkage and rehydration rate of samples with pretreatment are determined to compare with those of untreated samples. The results indicate that the drying rate of carrots chips can be improved by ultrasonic pretreatment, and $70 \mathrm{kHz}$ for pretreatment frequency, $45 \mathrm{~min}$ for pretreatment time and $25^{\circ} \mathrm{C}$ for pretreatment temperature is the optimum treated parameters.
\end{abstract}

Keywords--ultrasound pre-treatment; carrot; drying rate; frequency

\section{INTRODUCTION}

Drying dehydration becomes one of the important way to strategic food, because fresh fruits and vegetables have disadvantage of short of shelf life and transportation of import reagent products. Dried fruits and vegetables are more and more popular a mong people, which possesses the advantage of extending preservation time, long of shelf life and minimal loss of nutritional values. Drying dehydration plays an important role in food production process, especially in dried fruits and vegetables. Drying methods have: microwave drying and freeze-drying and so on, which has the shortcoming of high energy consumption and long drying time.

Ultrasound pre-treatment technology is often used in the application of food dehydration and production[1]. Ultrasonic sterilization and ultrasonic cleaning obtain the good effect. In all areas of ultrasonic, research and application of projects have been carried out which have reached the international advanced level. Ultrasound technology does not only have many magical effect, but also can be automated. There is a good prospect in the packaging industry. Ultrasonic pretreatment can improve the drying rate and reduce drying time, save energy and reduce product quality loss. The moisture in food can transfer liquid or gas out of food. Transfer processes are affected internal resistance and external resistance. Drying technology usually reduces mo isture migration resistance, ultras ound also can reduce water transfer resistance.

Ultrasound pre-treatment of food is a new technology which uses different frequencies to treat food for improving drying rate [2]. Yan Xiaohui et al[3] used ultrasound pre-treatment dealing with semi-dried litchi, the drying time reducing $10.87 \mathrm{~h}$. Kebitsamang Joseph Mothibe et al[4] used ultrasound pre-treatment dealing with dried fruits, the result showed that it does not only improve the drying rate but also reduces operation time. Ultrasonic pretreatment can reduce material moisture content, changing food material organization and improve the drying speed[5]. In 2004, some scholars studied the effect of fruit on air hot[6]. With the time changing, other scholars studied the ultras ound pre-treatment on fruit, J. X. Li et al[7] set a series of one-fact at a time experiments are conducted to investigate the effects of four process conditions including sucrose con centration, dehydration time, temperature, ultrasonic powder on water loss and solid gain, the optimal dehydration conditions obtained by response surface analysis are osmotic dehydration temperature of $65^{\circ} \mathrm{C}$, osmotic dehydration time of $2.46 \mathrm{~h}$, sucrose concentration of $56.29 \%$, and ultrasonic powder of $142.33 \mathrm{~W}$. In practice, these conditions are modified to be $65^{\circ} \mathrm{C}, 2.5,56 \%$ and $140 \mathrm{~W}$, respectively to obtain a water loss of $40.79 \%$ and a solid gain of $8.33 \%$ [7]. In many areas, a lot of scholars also studied ultrasound pre-treatment vegetables, fruit and so on, which showed that ultrasound pre-treatment can reduce drying time and increase drying rate [8-11].

China is the leading country of carrots production in the world, but carrots processing of frozen and fresh is in inferior position, which means there's some room for improvement [12]. Drying technology is one of the methods to maintain value of carrots[13]. Energy conservation is the trend of China's food industry, it is the 
development of research development and production process is simple, low energy consumption, green production. Researching and developing should be low short drying process, reduce energy consumption and product loss nutrition, while giving the product of good color and taste to achieve a win-win economic and social benefits[14]. The objective of this work is to study the effects of different ultrasound pre-treatment process parameters on the drying rate, shrinkage, rehydration ratio, water absorption and determine the optimal parameters of ultrasound pre-treatment on carrots.

\section{MATERIALS AND METHOD}

\section{A. Materials and equipment}

Fresh carrots are supplied from local supermarket. The ultrasound pre-treatment system is shown in Fig. 1, which provides adjustable frequency, temperature and time. Airdrying oven, electronic balance, culture dish, beaker, induction cooker and stainless steel kettle are used in the experiment.

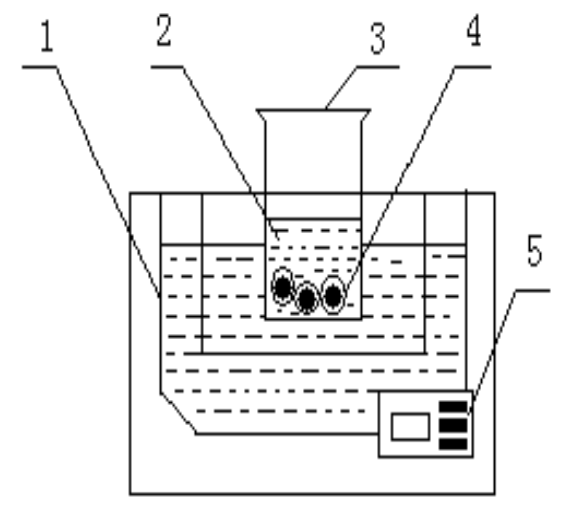

Figure 1. The ultrasound pre-treatment system. (1. Water channel 2. Water 3. Beaker 4. Carrots 5.Control power).

\section{B. Experiment Method}

There are three parameters of ultrasound pre-treatment processing, which are ultrasound frequency, time and temperature. According to these factors, different frequency $(50 \mathrm{hz}, 70 \mathrm{hz}, 100 \mathrm{hz})$, different time $(15 \mathrm{~min}$, $30 \mathrm{~min}, 45 \mathrm{~min})$, different temperature $\left(15^{\circ} \mathrm{C}, 25^{\circ} \mathrm{C}\right.$, $35^{\circ} \mathrm{C}$ ) are chosen to ultrasound pre-treatment carrots. And three blank groups and 30 experimental groups are set up among these experimental groups, 27 groups are chosen to ultrasound pre-treat at different factors and analyzing the optimal parameters. Using the optimal parameters to pretreat other 3 experimental groups and comparing with 3 blank groups. Finally, the effect of ultrasonic pretreatment to shrinkage, rehydration ratio and water absorption can be achieved.

\section{Pre-treatment}

The carrots are washed and cut into 33 groups which are $4 \mathrm{~mm}$ thick slices, the same weight and volume. All of carrots recorded the weight and volume. Three groups of carrots are untreated as blank groups, 27 experimental groups are ultrasound treated in different frequency $(50 \mathrm{hz}$, $70 \mathrm{hz}, 100 \mathrm{hz})$, different time (15 min, 30min, 45min), different temperature $\left(15^{\circ} \mathrm{C}, 25^{\circ} \mathrm{C}, 35^{\circ} \mathrm{C}\right)$, respectively. Other 3 experimental groups are treated at optimal parameters. Finally, measure drying time, shrinkage, rehydration ratio and water absorption.

\section{Shrinkage Procedure}

The volume can be reflected during the drying process, record the volume before drying. When carrots are treated by ultrasound, measure the volume of carrots, subtracting and dividing the original volume to make sure shrinkage.

\section{E. Rehydration ratio measurment}

Rehydration ratio measures should be in hot water at $100^{\circ} \mathrm{C}$, when saturated, took them out and drained them. Recorded the weight of carrots, which is calculated as rehydration ratio.

\section{F. Water absorption measurement}

Soak the dried carrots into water at $20^{\circ}$ C. Drain the carrots after an hour, record and weight, which is calculated as water absorption.

\section{G. Indexess}

$$
\begin{gathered}
\text { Drying rate }=\left(M-M_{d}\right) T^{-1} \times 100 \% \\
\text { Shrinkage }=\left(V_{0}-V_{f}\right) V_{0}^{-1} \times 100 \% \\
\text { Rehydration ratio }=\left(M_{f}-M_{g}\right) M_{g}{ }^{-1} \times 100 \%
\end{gathered}
$$

$$
\text { Water absorption }=\left(M_{d} \cdot M_{s}^{-1}\right) \times 100 \%
$$

$\mathrm{M}$ is initial weight $(\mathrm{g})$, Md is drying weight $(\mathrm{g}), \mathrm{T}$ is drying time $(\mathrm{min}), \mathrm{V} 0$ is the volume of not drying $(\mathrm{cm} 3)$, $\mathrm{Vf}$ is the volume of drying ( $\mathrm{cm} 3)$, Mf is the weight of not soaked $(\mathrm{g}), \mathrm{Mg}$ is the weight of drained $(\mathrm{g})$. Ms is the weight of soaked at $20^{\circ} \mathrm{C}$.

\section{RESULTS AND DISCUSSION}

\section{A. Effect of different Ultrasound pretreatment frequency, time and temperature on drying rate}

Fig. 2 presents the effect of the three different pretreatment frequency, temperature and time on drying rate. Three graphs of (a), (b) and (c) with increasing frequency, the drying rate dropped after the first rise, when the maximum drying rate is frequency of $70 \mathrm{hz}$ and drying time of $45 \mathrm{~min}$. Other three graphs of (d), (e) and (f), drying rate has the biggest numerical at $25^{\circ} \mathrm{C}$. The results shows that the drying rate of ultrasound pre-treatment sample is improved obviously. Under the circumstance of $70 \mathrm{~Hz}$, $45 \mathrm{~min}$ and $25^{\circ} \mathrm{C}$, the drying rate of pretreatment is most 
highest than that of control groups, the best drying rate is $5.76 \mathrm{~g} / \mathrm{h}$

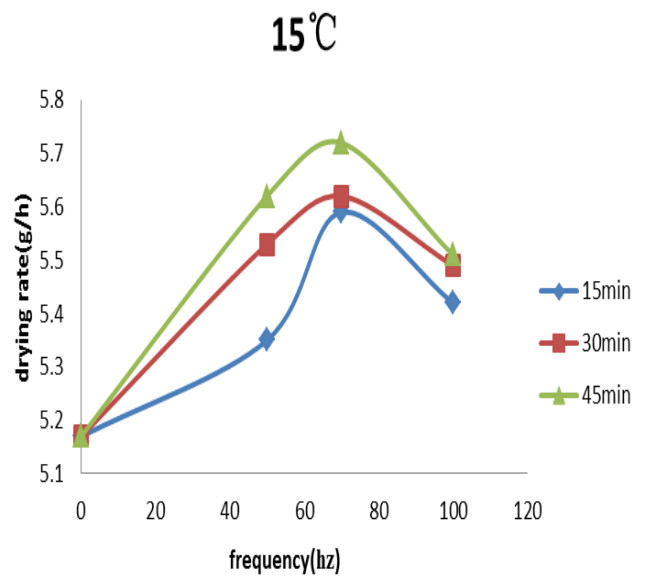

(a)

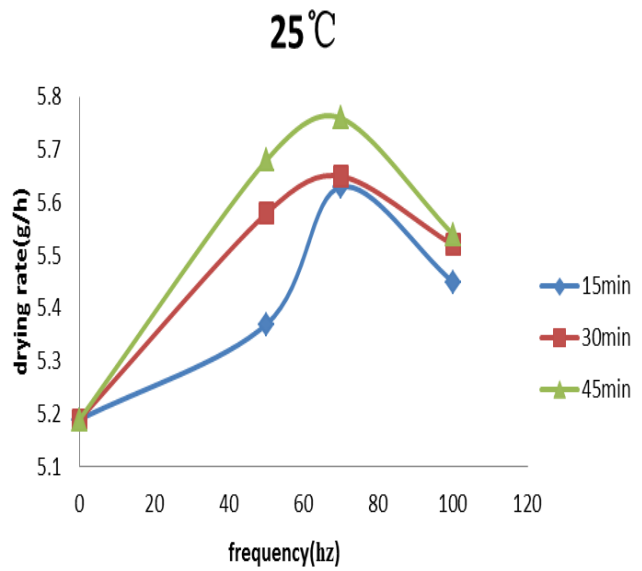

(b)

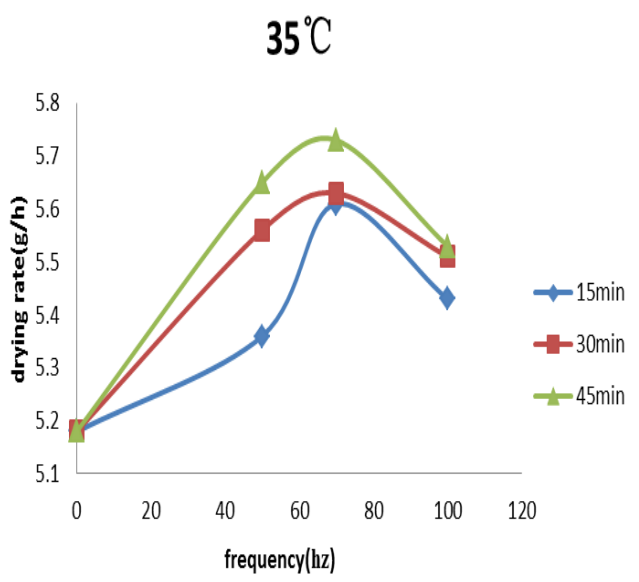

(c)
$70 \mathrm{hz}$

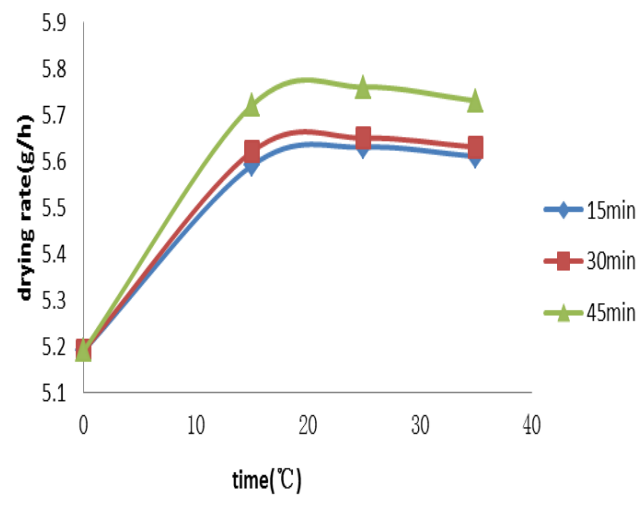

(d)

$70 \mathrm{hz}$

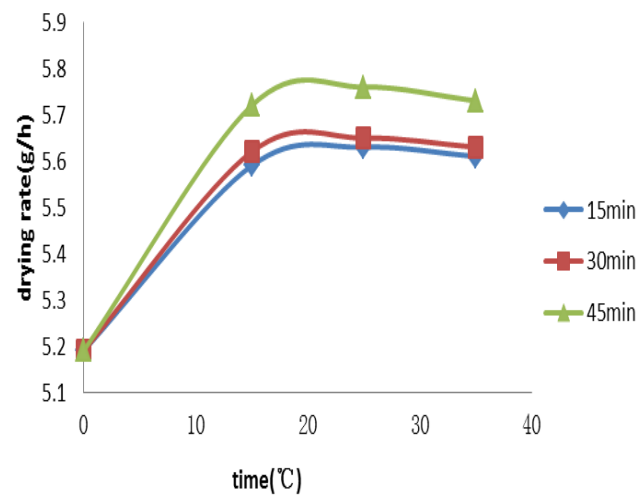

(e)

\section{$100 \mathrm{hz}$}

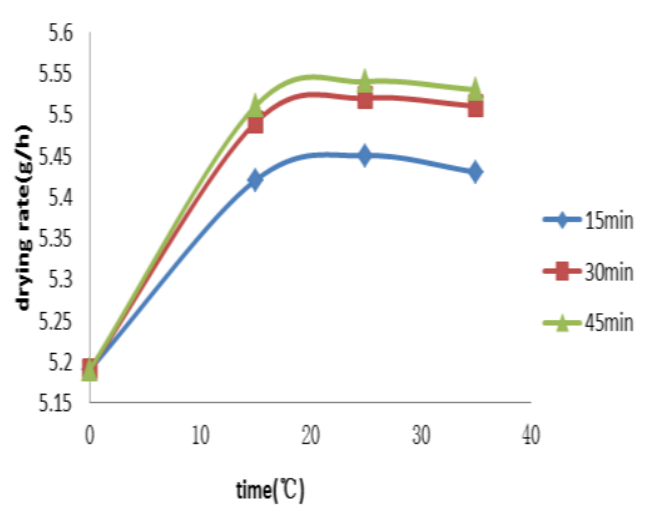

(f)

Figure 2. Six graphs of drying rate against pre-treatment frequency, temperature and time

\section{B. Effect of Ultrasound pre-treatment on the Shrinkage}

Fig. 3 shows the results of shrinkage of carrots. The volume of untreated fresh carrot is $1.81 \mathrm{~cm} 3$, when it dried by hot air the volume decrease to $0.65 \mathrm{~cm} 3$. Ultrasound pre-treatment fresh carrot volume is $1.79 \mathrm{~cm} 3$, when it is dried by hot air the volume become to $0.63 \mathrm{~cm} 3$. The shrinkage of pretreated samples is $65.3 \%$, and shrinkage 
untreated samples is $64.1 \%$. Therefore, ultrasound pretreatment has no apparent influence on shrinkage.

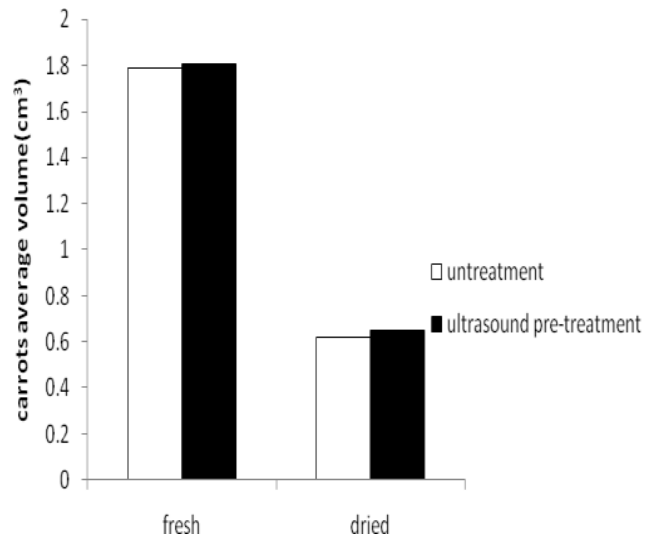

Figure 3. Effect of ultrasound pretreatment on volume change of shrinkage

\section{Effect of Ultrasound pre-treatment on the rehydration ratio}

Fig. 4 shows the effect of ultras ound pretreatments on the rehydration ratio. The weight of untreated drying carrot is $0.47 \mathrm{~g}$, when it saturates at $100^{\circ} \mathrm{C}$ water and drains it, the volume of carrot is $4.81 \mathrm{~g}$. Ultrasound pre-treatment carrot's weight vary $0.43 \mathrm{~g}$ to $0.71 \mathrm{~g}$. The rehydration ratio of pretreatments is 0.08 times higher than untreated. Therefore, ultrasound pre-treatment has little impact on rehydration rate of carrots.

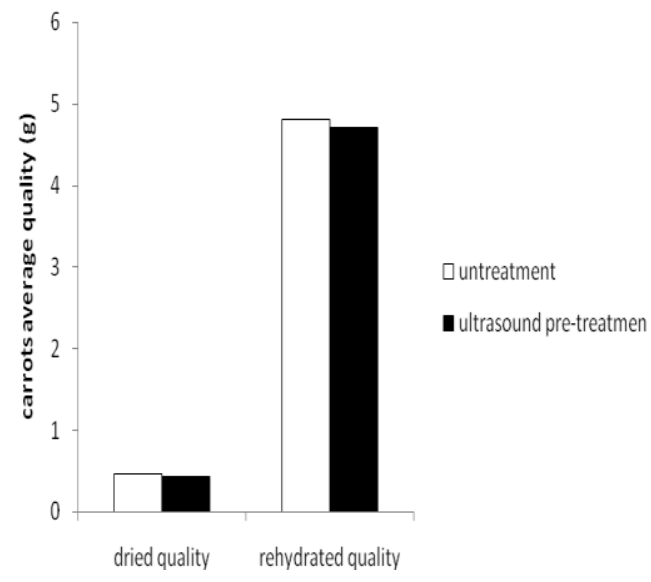

Figure 4. Effect of ultrasound pretreatment on volume change of shrinkage

\section{Effect of Ultrasound pre-treatment on the Water Absorption}

The influence of ultrasound pretreatment on the water absorption is shown in Fig. 5 The weight of untreated carrot is $0.47 \mathrm{~g}$ and ultrasound pre-treatment carrot is $0.41 \mathrm{~g}$. When them absorbed in water, the weight becomes $4.81 \mathrm{~g}$ and $4.45 \mathrm{~g}$. By calculating, the water absorption of pretreatments is 0.067 times higher than untreated. Therefore, ultrasound pre-treatment has little effect on water absorption.

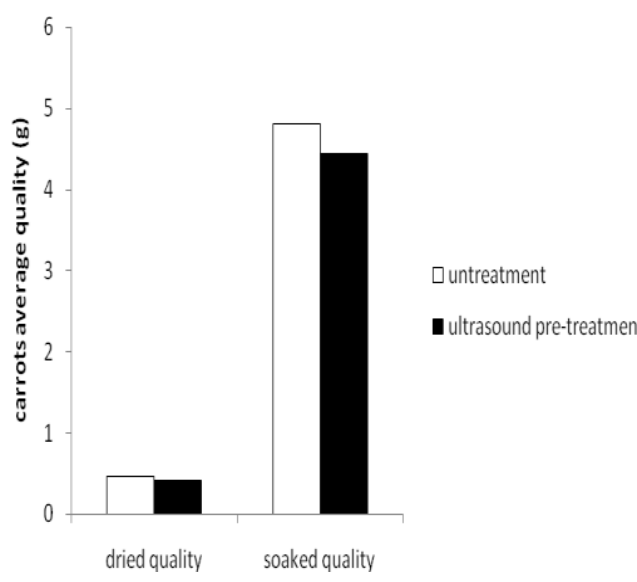

Figure 5. Effect of ult rasound pretreatment on quality change of water absorption

\section{CONCLUSIONS}

The drying rate of carrots can be improved by ultrasound pretreatment. Drying rate with the growth of drying time increases, the effect is best when drying time is $45 \mathrm{~min}$. And the optimum treated parameters are as follows: pretreatment temperature is $25^{\circ} \mathrm{C}$, pretreatment frequency is $70 \mathrm{~Hz}$ and pretreatment time is $45 \mathrm{~min}$. The ultrasound pretreatment has no effect on the quality of carrots.

\section{REFERENCES}

[1] F. Zhao, Z. Q. Chen, M. H. Shi, "Experimental study on mass transfer process in ultrasonic pre-dehydration of apple slice," Jounal of southeast university. J. China, vol. 41, pp. 124-128, January 2011.

[2] D. L. Luo, B. C. Xu, W. X. Zhu, J. X. Liu, "Study and progress of power ultrasound in hot-air drying area," Journal of the Cereals and Oils Assoctation. J. China, vol. 28, pp. 123-128, March 2013.

[3] X. H. Yan, X. L. Yu, H. Z. Yan, K. Yu, Y. L. Zheng and Y. J. Li, "Effects of ultrasound pretreatment on drying time for intermediate-dried litchi," Transactions of the CSAE. J. Chnia, vol. 27 , pp. 351-356, March 2011.

[4] G. N. Juan, I. P .O. Francisca, I. G. Maria, L. W. Curtis, R. Sueli, A. N. F. Fabiano, "Ultrasound- Assisted Osmotic Dehydration of Strawberries: Effect of Pretreatment Time and Ultrasonic Frequency," Drying Technology. J. London, vol. 28, pp.294-303, 2010.

[5] L. F. Zeng, "Application of Ultrasonic in Food stuff Drying," www. gdchem. Com. J. China, vol. 35, pp. 49-51, April 2008.

[6] P. A. Zhang, S. Y. Ye, Y. Li, W. Huang, S. C. Luo, "Study on osmotic dehydration of longan sarcocarp enhanced by ultrasound field," Journal of South China Agricultural University (Natural Science Edition). J. China, vol25, pp. 101-103, April 2004.

[7] J. X. Li, Y. Zhang, Q. Dong, "Optimization of UltrasonicIntensified Osmotic Dehydration of Purple Sweet Potato Slices," Food Science. J. China. Vol33, pp 73-77, July 2012.

[8] Kehinde. A. T, Mohammad. N. E, Beatrice. I. O, "Osmotic dehydration of strawberry halves: influence of osmotic agents and pretreatment methods on mass transfer and product characteristics" International Journal of Food Science and Technology. J. China, vol 38, pp693-707,2003

[9] C. Q. Zhao, Z. F. Wang, X. S. Hu, "Effects of ultrasonic pre treatment on osmotic dehydration of cherry tomato," Science and Technology of Food Indrstry. J. China, vol29, pp 149-150, March 2008 
[10] X. E. Ren, R. He, Y. C. Huang, J. M. Zhang, F.Yang, "Osmotic Dehydration of Pineapple Enhanced by Ultrasonic Treatment," Food Science. J. China, vol31, pp 279-282, November 2010

[11] C. Zeng, J. F. Bi, J. Pang, "Research Progress of Explosion Puffinig Drying Technology of Carrot at Variable Temperat ure and Pressure Difference," Food Science. J. China, vol29, pp. 756-759, December 2008.

[12] J. V. García-Pérez, J. A. Cárcel, J. Benedito, et al. "Drying of a low porosity product (carrot) sa affected by power ultrasound" $3 \mathrm{rd}$ International Conference on Diffusion in Solids and Liquids. Algarve, Portugal, 2007,pp764-769.
[13] X. X. Wu, H. Y. Zhang, W. H. Zhang, K. J.Ma, "The osmotic dehydration of white radish enhanced by ultrasound field," Food science and technology. J. China, vol38, pp. 112-115, May 2013G. Eason, B. Noble, and I. N. Sneddon, "On certain integrals of Lipschitz-Hankel type involving products of Bessel functions," Phil. Trans. Roy. Soc. London, vol. A247, pp. 529-551, April 1955. (references)

[14] W. R. Li, R. A. Ren, G. B. Chen, "Research Progress of Drying Technology of carrots," Modern Agricultural Science and Technology. J. China, pp. 388-390, July. 2010. 